\title{
Highly Efficient Green Light-Emitting Diodes from All-Inorganic Perovskite Nanocrystals Enabled by a New Electron Transport Layer
}

\author{
Baiquan Liu, Lin Wang, Haoshuang Gu,* Handong Sun,* and Hilmi Volkan Demir*
}

Adopting proper electron transport layers (ETLs) is essential to high-performance all-inorganic perovskite light-emitting diodes (PeLEDs). However, the effect of ETLs has not been comprehensively investigated in all-inorganic nanocrystal PeLEDs, while 2,2',2"'-(1,3,5-benzenetriyl) tris-[1-phenyl-1H-benzimidazole] (TPBi) is the most common ETL. Herein, a novel strategy is proposed to enhance the efficiency of nanocrystal PeLEDs. Tris(8-hydroxyquinoline) aluminum $\left(\mathrm{Alq}_{3}\right)$ is incorporated into TPBi to form a new ETL TPBi/ $\mathrm{Alq}_{3} / \mathrm{TPBi}$, simultaneously enabling charge balance and confinement. The green PeLED with new ETL exhibits a maximum external quantum efficiency (EQE) of $1.43 \%$, current efficiency of $4.69 \mathrm{~cd} \mathrm{~A}^{-1}$, and power efficiency of $1.84 \mathrm{~lm} \mathrm{~W}^{-1}$, which are $191 \%, 192 \%$, and $211 \%$ higher than those of PeLEDs with conventional ETL TPBi, respectively. Significantly, the EQE is 36-fold higher than that of PeLED with high electron mobility ETL. Impressively, the full width at half-maximum of electroluminescence emission is $16 \mathrm{~nm}$, which is the narrowest among $\mathrm{CsPbBr}_{3}$ PeLEDs. The findings may present a rational strategy to enhance the device engineering of all-inorganic PeLEDs.

Lead halide perovskites have recently emerged as a new family of optoelectronic materials for applications including solar cells, lasers, and photodetectors because of their impressive characteristics including narrow emission, size-tunable optical bandgaps,

Dr. B. Liu, Prof. H. V. Demir

Luminous! Center of Excellence for Semiconductor Lighting and Displays

School of Electrical and Electronic Engineering and

School of Physical and Mathematical Sciences

Nanyang Technological University

Singapore 639798, Singapore

E-mail: hvdemir@ntu.edu.sg

L. Wang, Prof. H. Sun

Division of Physics and Applied Physics

School of Physical and Mathematical Sciences

Nanyang Technological University

Singapore 637371, Singapore

E-mail: hdsun@ntu.edu.sg

L. Wang, Prof. H. Gu

Hubei Collaborative Innovation Center for Advanced Organic

Chemical Materials

Hubei Key Laboratory of Ferro \& Piezoelectric Materials and Devices

Faculty of Physics \& Electronic Sciences

Hubei University

Wuhan 430062, China

E-mail: guhsh@hubu.edu.cn

The ORCID identification number(s) for the author(s) of this article can be found under https://doi.org/10.1002/adom.201800220. and excellent charge-transport capabilities. ${ }^{[1-5]}$ These exceptional properties have also made perovskites suitable for applying to light-emitting diodes (LEDs). ${ }^{[6]}$ Since the first demonstration of hybrid organicinorganic $\mathrm{CH}_{3} \mathrm{NH}_{3} \mathrm{PbBr}_{3}$ perovskite LED (PeLED) in 2014,[7] PeLEDs have rapidly attracted a great deal of attention from both academic and industrial researchers. ${ }^{[8-10]}$ So far, the highest external quantum efficiency (EQE) for hybrid organic-inorganic PeLED can reach $11 \% .{ }^{[11]}$ However, hybrid organicinorganic perovskite materials suffer from the stability issue, which is a hurdle for the widespread use. Alternatively, all-inorganic perovskites (e.g., $\mathrm{CsPbX}_{3}, \mathrm{X}=\mathrm{I}, \mathrm{Br}$, and $\mathrm{Cl}$ or mixed halide) possess superior thermal stability than their hybrid counterparts. ${ }^{[12-14]}$ Besides, all-inorganic perovskites can exhibit high photoluminescence quantum yield (PLQY, e.g., > 90\% in solution) and narrow emissions (e.g., full width at halfmaximum (FWHM) $<30 \mathrm{~nm}$ ), and are compatible with the solution processing technology, which triggers intense interest in applying all-inorganic perovskites to develop LEDs since the first report of all-inorganic PeLED with a maximum EQE of $0.12 \%$ by

DOI: 10.1002/adom.201800220

Prof. H. Sun

Centre for Disruptive Photonic Technologies (CDPT)

School of Physical and Mathematical Sciences

Nanyang Technological University

21 Nanyang Link, Singapore 637371, Singapore

Prof. H. Sun

MajuLab

CNRS-UCA-SU-NUS-NTU International Joint Research Unit

Singapore

Prof. H. V. Demir

Department of Electrical and Electronics Engineering

Department of Physics

UNAM-Institute of Materials Science and Nanotechnology

Bilkent University

Ankara 06800, Turkey 
Zeng and co-workers in 2015. ${ }^{[15]}$ Moreover, these characters make them even very promising for solution-processible lasers. ${ }^{[16-18]}$

Over the past two years, a large number of approaches have been reported to improve the performance of all-inorganic PeLEDs, such as using simple trimethylaluminum crosslinking technique to render the nanocrystal films insoluble, ${ }^{[19]}$ passivating $\mathrm{CsPbX}_{3}$ quantum dots (QDs) with halide and mixed halide ion pairs via a two-step ligand exchange process, ${ }^{[20]}$ forming phase-stable QD films to obtain device with low turnon voltage and tunable emission, ${ }^{[21]}$ and balancing surface passivation and carrier injection via ligand density control. ${ }^{[22]}$ To achieve the high performance, it is noted that most of the reports about all-inorganic PeLEDs are mainly focused on the improvement of emitting materials $\mathrm{CsPD}_{3}$, and their performance has been gradually improved. ${ }^{[19-25]}$

Apart from the optimizations of emitting materials $\mathrm{CsPbX}_{3}$, the innovation of device engineering is an alternative and even more critical way to boost the performance of nanocrystal allinorganic PeLEDs. ${ }^{[26]}$ However, the effort in device engineering lags far behind relative to the improvement of emitting materials. For example, Rogach and co-workers introduced a thin film of perfluorinated ionomer sandwiched between the hole transport layer (HTL) and perovskite emissive layer (EML) to improve the hole injection of $\mathrm{CsPbBr}_{3}$ PeLEDs, achieving an EQE of $0.06 \% .{ }^{[27]}$ Besides, they used an insulating material polyhedral oligomeric silsesquioxane as a solution additive or an additional hole-blocking layer to improve the performance of $\mathrm{CsPBr}_{3}$ PeLEDs, achieving an EQE of $0.35 \% .{ }^{[28]}$ Therefore, it has been a major challenge to develop high-efficiency nanocrystal allinorganic PeLEDs via the enhancement of device engineering, although the reported methods can improve the efficiency.

In nanocrystal all-inorganic PeLEDs, adopting suitable electron transport layers (ETLs) is essential to realizing high performance. This is because the charge transport, charge leakage, and charge balance can be largely affected by ETLs. However, the effect of ETLs has not yet been comprehensively investigated. So far, 2,2',2'-(1,3,5-benzenetriyl) tris-[1-phenyl-1H-benzimidazole] (TPBi) is the most widely used ETL in all-inorganic PeLEDs, ${ }^{[15,19-25,27-29]}$ and there is no report for any other ETL that can outperform TPBi in all-inorganic PeLEDs.

In this paper, a novel strategy has been proposed to enhance the device engineering of nanocrystal all-inorganic PeLEDs employing tris(8-hydroxyquinoline) aluminum ( $\left.\mathrm{Alq}_{3}\right)$ into TPBi to form a new TPBi/Alq 3 /TPBi ETL, which enables the charge balance and charge confinement simultaneously. The green PeLED with the new ETL TPBi/Alq $/$ TPBi can exhibit a maximum EQE, current efficiency (CE), and power efficiency (PE) of $1.43 \%, 4.69 \mathrm{~cd} \mathrm{~A}^{-1}$, and $1.84 \mathrm{~lm} \mathrm{~W}^{-1}$, respectively, which are correspondingly $191 \%, 192 \%$, and $211 \%$ higher than those of the PeLED with the conventional ETL TPBi. Importantly, the efficiency of green PeLED with the new ETL is 36 times higher than that of PeLED with high electron mobility based ETL, further indicating the advantage of this novel strategy. Simultaneously, the FWHM of the electroluminescence (EL) emission is only $16 \mathrm{~nm}$, which is the narrowest value among

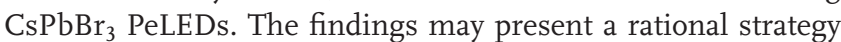
to enhance the device engineering of all-inorganic PeLEDs.

To appropriately explore the effect of ETLs in nanocrystal allinorganic PeLEDs, several tactics have been employed. First, it is necessary to guarantee that there are enough holes that can bound with electrons to form excitons, otherwise lack of holes will have a negative influence on device performance. ${ }^{[30]}$ Therefore, we employed poly(9-vinlycarbazole) (PVK) as the HTL, of which the highest occupied molecular orbital (HOMO) is $5.6 \mathrm{eV},{ }^{[28]}$ located between the hole injection layer poly(ethylen edioxythiophene):polystyrene sulfonate (PEDOT:PSS) and EML $\mathrm{Cs} \mathrm{PbBr}_{3}$, which can reduce the hole injection barrier. Besides, the lowest unoccupied molecular orbital of PVK is $2.2 \mathrm{eV}$, much higher than the conduction band of $\mathrm{CsPbBr}_{3}(3.8 \mathrm{eV})$, which can effectively confine electrons in the active layer. ${ }^{[28]}$ As a result, more holes and electrons can be expected to recombine in the EML. Then, three typical electron transport materials $\mathrm{Alq}_{3}$, TPBi, and 4,7-diphenyl-1,10-phenanthroline (Bphen) have been first selected as the ETLs of green PeLEDs. The reason why they have been chosen is that they have different electron mobility and HOMO, readily to investigate the effects of ETL on charge transport and charge confinement. Particularly, since TPBi is the most popular ETL in all-inorganic PeLEDs, the comparison between TPBi and other ETLs is significant to optimize ETLs. Besides, due to the excellent properties, $\mathrm{Alq}_{3}$ has been heavily explored in organic light-emitting diode (OLED), ${ }^{[31-34]}$ and other applications such as nonvolatile random access memory, ${ }^{[35]}$ magnetoresistance applications, ${ }^{[36]}$ quantum optics devices, ${ }^{[37]}$ and molecular spintronics devices. ${ }^{[38]}$ However, $\mathrm{Alq}_{3}$ has not yet been explored in all-inorganic PeLEDs to date. In addition, we fixed the ETL thickness at $35 \mathrm{~nm}$, since additional reductions in efficiency could occur when the thickness of the ETL is too thin (e.g., $<25 \mathrm{~nm}$ ) due to exciton quenching at the metal cathode. ${ }^{[39]}$ Finally, to greatly enhance the efficiency of nanocrystal all-inorganic green PeLEDs, a new ETL TPBi/Alq $\mathrm{A}_{3} /$ TPBi has been proposed.

Taking the above factors into account, here we present a systematic investigation on the effect of ETL in all-inorganic PeLEDs. As shown in Figure 1, the structure of green PeLEDs is indium tin oxide (ITO)/PEDOT:PSS (40 nm)/PVK $(10 \mathrm{~nm}) / \mathrm{CsPbBr}_{3}\left(20 \mathrm{~nm}, 10 \mathrm{mg} \mathrm{mL}{ }^{-1}, 2000 \mathrm{rpm}, 45 \mathrm{~s}\right) / \mathrm{ETLs}$ $(35 \mathrm{~nm}) / \mathrm{Cs}_{2} \mathrm{CO}_{3}(1 \mathrm{~nm}) / \mathrm{Al}(100 \mathrm{~nm})$, where ITO is the anode, PEDOT:PSS is the hole injection layer, PVK is the HTL, $\mathrm{Cs}_{2} \mathrm{CO}_{3}$ is the electron injection layer, $\mathrm{Al}$ is the cathode, and ETLs are $\mathrm{Alq}_{3}$, TPBi, and Bphen for Devices G1, G2, and G3, respectively. The PLQY of $\mathrm{CsPbBr}_{3}$ is as high as $90 \%$. The absorption and steady-state PL characteristics of $\mathrm{CsPbr}_{3}$ in hexane at room temperature are shown in Figure S1 (Supporting Information), the scanning transmission electron microscopy image of $\mathrm{CsPBr}_{3}$ is displayed in Figure S2 (Supporting Information), and the cross-sectional scanning electron microscope image of the PeLED is depicted in Figure S3 (Supporting Information).

As shown in Figure 2 and Table S1 (Supporting Information), the maximum EQE of Device G2 is $0.75 \%$, which is higher than those of Devices G1 (0.73\%) and G3 (0.04\%). Similarly, the maximum CE of Device G2 is $2.44 \mathrm{~cd} \mathrm{~A}^{-1}$, higher than those of Devices G1 (2.36 cd A $\mathrm{A}^{-1}$ ) and G3 (0.15 $\left.\mathrm{cd} \mathrm{A}^{-1}\right)$. Besides, the maximum PE of Device $\mathrm{G} 2$ is $0.87 \mathrm{~lm} \mathrm{~W}^{-1}$, higher than those of Devices G1 $\left(0.84 \mathrm{~lm} \mathrm{~W}^{-1}\right)$ and G3 $\left(0.05 \mathrm{~lm} \mathrm{~W}^{-1}\right)$. Since all layers in these PeLEDs are similar except for the ETL, the EL properties suggest that TPBi is more favorable to ensure the high efficiency. Furthermore, compared to the EL spectra of Devices G2 and G3, the spectrum of Device G1 is much broader, as shown 
(a)

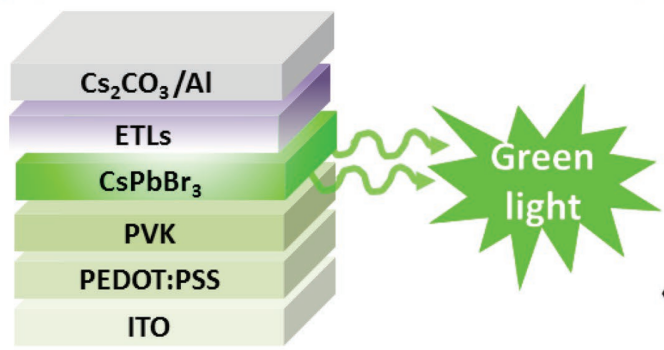

(c)
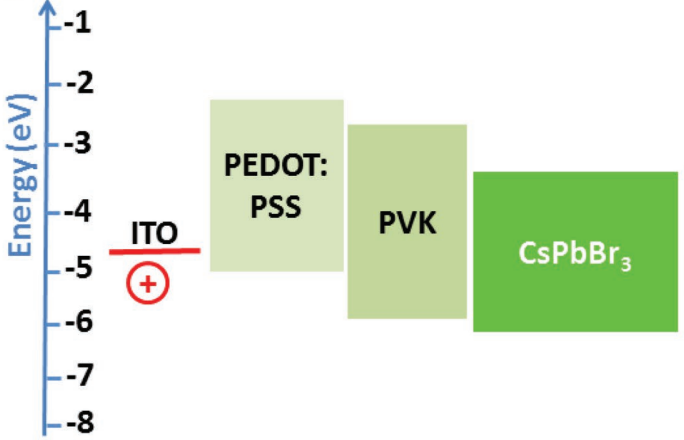

(b)

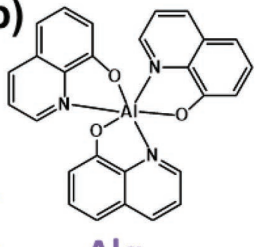

$\mathrm{Alq}_{3}$

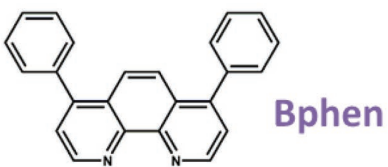

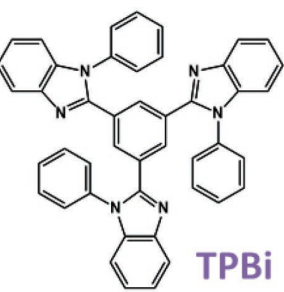

TPBi
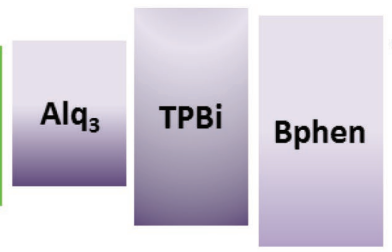

$\mathrm{Cs}_{2} \mathrm{CO}_{3} / \mathrm{Al}$

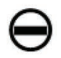

$\theta$

Figure 1. a) The schematic structure of PeLEDs based on $\mathrm{CsPbBr}_{3}$. b) The chemical structure of ETLs. c) The proposed energy levels of the PeLEDs based on $\mathrm{CsPbBr}_{3}$.

in Figure 2d. In addition, the emission peak of Device G1 is $518 \mathrm{~nm}$, which is different with those of Devices G2 (516 nm) and G3 $(516 \mathrm{~nm})$. Therefore, the spectrum of Device G1 cannot be attributed to the only $\mathrm{CsPbBr}_{3}$ emission.
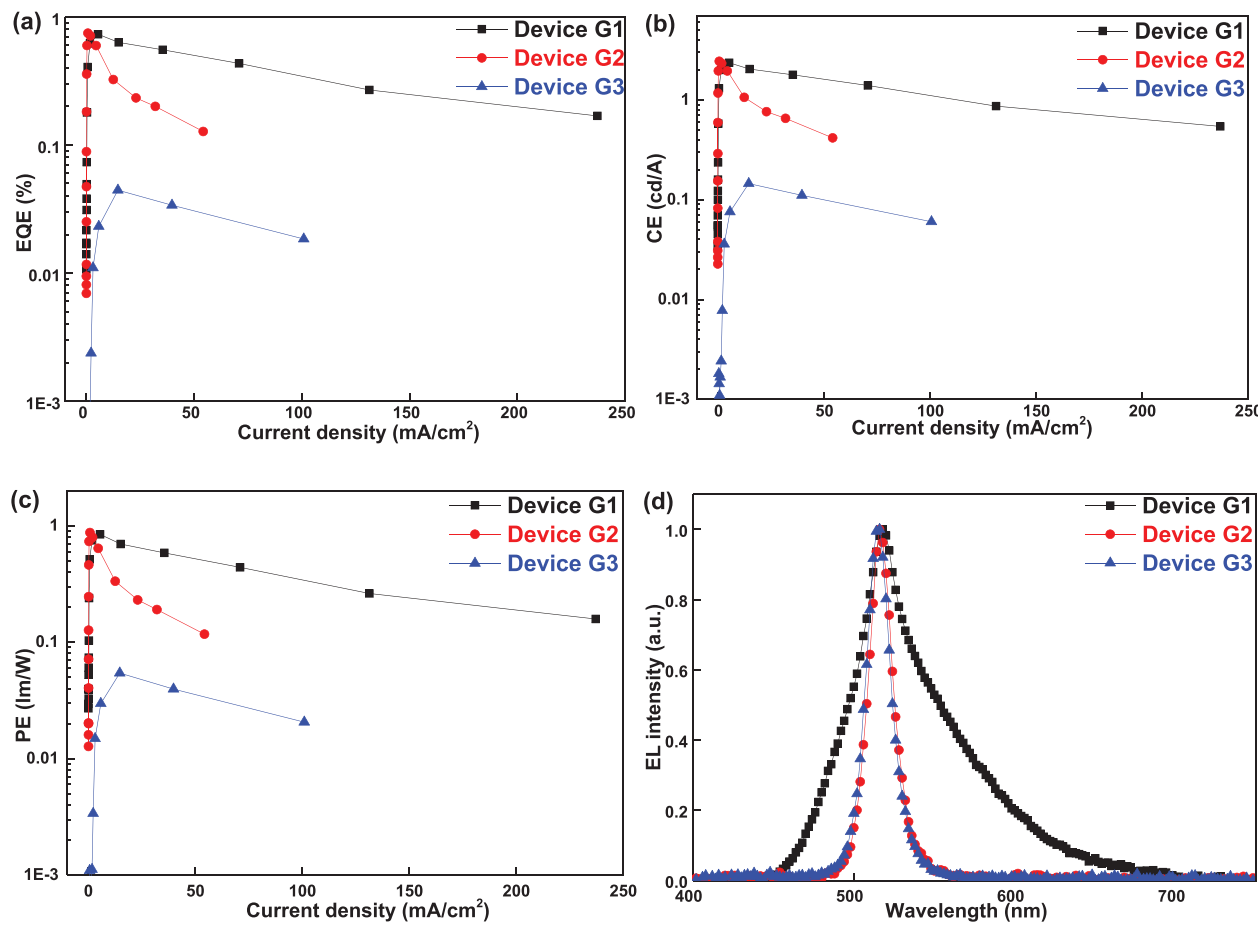

Figure 2. a) EQE as a function of the current density for Devices G1, G2, and G3. b) CE of Devices G1, G2, and G3. c) PE of Devices G1, G2, and G3. d) EL spectra of Devices G1, G2, and G3 at $8.5 \mathrm{~V}$. 
efficiency play critical roles in LEDs. ${ }^{[40]}$ When contacting with the typical electron injection layer $\mathrm{Cs}_{2} \mathrm{CO}_{3}$ or $\mathrm{LiF}$, it has been well demonstrated that all of $\mathrm{Al}_{\mathrm{q}_{3}}, \mathrm{TPBi}$, and Bphen can show high electron injection efficiency. ${ }^{[40,41]}$ Thus, the electron injection efficiency has little influence on these PeLEDs. On the other hand, the electron mobility of $\mathrm{Alq}_{3}, \mathrm{TPBi}$, and Bphen are $1.4 \times 10^{-6}, 3.3 \times 10^{-5}$, and $3.9 \times 10^{-4} \mathrm{~cm}^{2} \mathrm{~V}^{-1} \mathrm{~s}^{-1}$, respectively. ${ }^{[41]}$ Hence, the electron mobility of $\mathrm{Alq}_{3}$ is almost equal to the hole mobility of PVK $\left(1.0 \times 10^{-6} \mathrm{~cm}^{2} \mathrm{~V}^{-1} \mathrm{~s}^{-1}\right)$, ${ }^{[22]}$ while the electron mobility of TPBi and Bphen is much higher than the hole mobility of PVK. In LEDs, the EQE is defined as follows ${ }^{[42]}$

$\mathrm{EQE}=\eta_{\text {out }} r q \gamma$

where $\eta_{\text {out }}$ is the outcoupling factor, $r$ is the fraction of excitons that can potentially radiatively decay, $q$ is the PLQY of emitters, and $\gamma$ is the charge balance $(\gamma \leq 1)$. Generally, the internal operation of LEDs does not influence $\eta_{\text {out }}$, while $r$ and $q$ would be set with emitters. Hence, from the perspective of device engineering, the EQE is the most sensitive to $\gamma$. In terms of charge mobility, Device G3 should exhibit the lowest efficiency since its charge balance is the worst among these devices (i.e., the electron mobility of Bphen is 2 orders of magnitude higher than the hole mobility of PVK), while Device G1 should exhibit the highest efficiency since the electron mobility of $\mathrm{Alq}_{3}$ is almost equal to the hole mobility of PVK. As expected, Device G3 exhibits the poorest efficiency. However, the highest EQE is achieved in Device G2 instead of Device G1, although the electron mobility of TPBi is much higher than the hole mobility of PVK. Therefore, aside from the charge mobility, other properties of ETLs should also greatly affect the resulting PeLEDs.
In the case of hole confining, the $\mathrm{HOMO}$ of $\mathrm{Alq}_{3}$ is $5.9 \mathrm{eV}$, which is higher than the valance band (VB) of $\mathrm{CsPbBr}_{3} \cdot{ }^{[40]} \mathrm{On}$ the other hand, the HOMO of TPBi $(6.2 \mathrm{eV})$ and Bphen $(6.4 \mathrm{eV})$ is lower than the $\mathrm{VB}$ of $\mathrm{CsPbBr}_{3} \cdot{ }^{[43]} \mathrm{As}$ a result, the $\mathrm{Alq}_{3}$ ETL is unable to confine the holes in the $\mathrm{CsPbBr}$ EML, leading to the hole leakage, while TPBi and Bphen are more favorable to confine the holes. Since $\mathrm{Al}_{3}$ is a well-known emitter in OLEDs, ${ }^{[44]}$ the leaked holes from the $\mathrm{CsPBBr}_{3}$ EML can meet electrons in the $\mathrm{Alq}_{3}$ ETL to generate excitons and then the excitons can recombine for the $\mathrm{Alq}_{3}$ emission, as shown in Figure 3a. Due to the participation of $\mathrm{Alq}_{3}$ emission, it is no wonder that the EL spectrum of Device G1 is much wider than those of Devices G2 and G3. These analyses can be further verified by comparing the EL spectrum of Device $\mathrm{G} 1$ and $\mathrm{Alq}_{3}$ emission, as shown in Figure S4 (Supporting Information). Therefore, the charge confining capability of ETLs is significant in PeLEDs.

Since the charge leakage is detrimental to the performance, ${ }^{[28]}$ Device G1 exhibits lower efficiency than Device G2, although the electron mobility of TPBi is much higher than the hole mobility of PVK (Figure 3b). On the other hand, despite Bphen possesses excellent hole confining capability, excess electrons can reach the $\mathrm{CsPbBr}_{3}$ EML due to the high electron mobility and good electron injection efficiency of Bphen, leading to a very poor charge balance in Device G3, as shown in Figure 3c. Thus, according to Equation (1), it is reasonable that Device G3 exhibits a low efficiency. Although Device G2 exhibits the highest efficiency among the achieved devices due to the use of TPBi ETL, its charge balance is still not good enough (Figure $3 \mathrm{~b}$ ). To further enhance the charge balance, $\mathrm{Alq}_{3}$ has been inserted into TPBi to form a new ETL TPBi $(15 \mathrm{~nm}) / \mathrm{Alq}_{3}(10 \mathrm{~nm}) / \mathrm{TPBi}(10 \mathrm{~nm})$ to fabricate Device G4 (other layers are the same as those of Device G2). Since
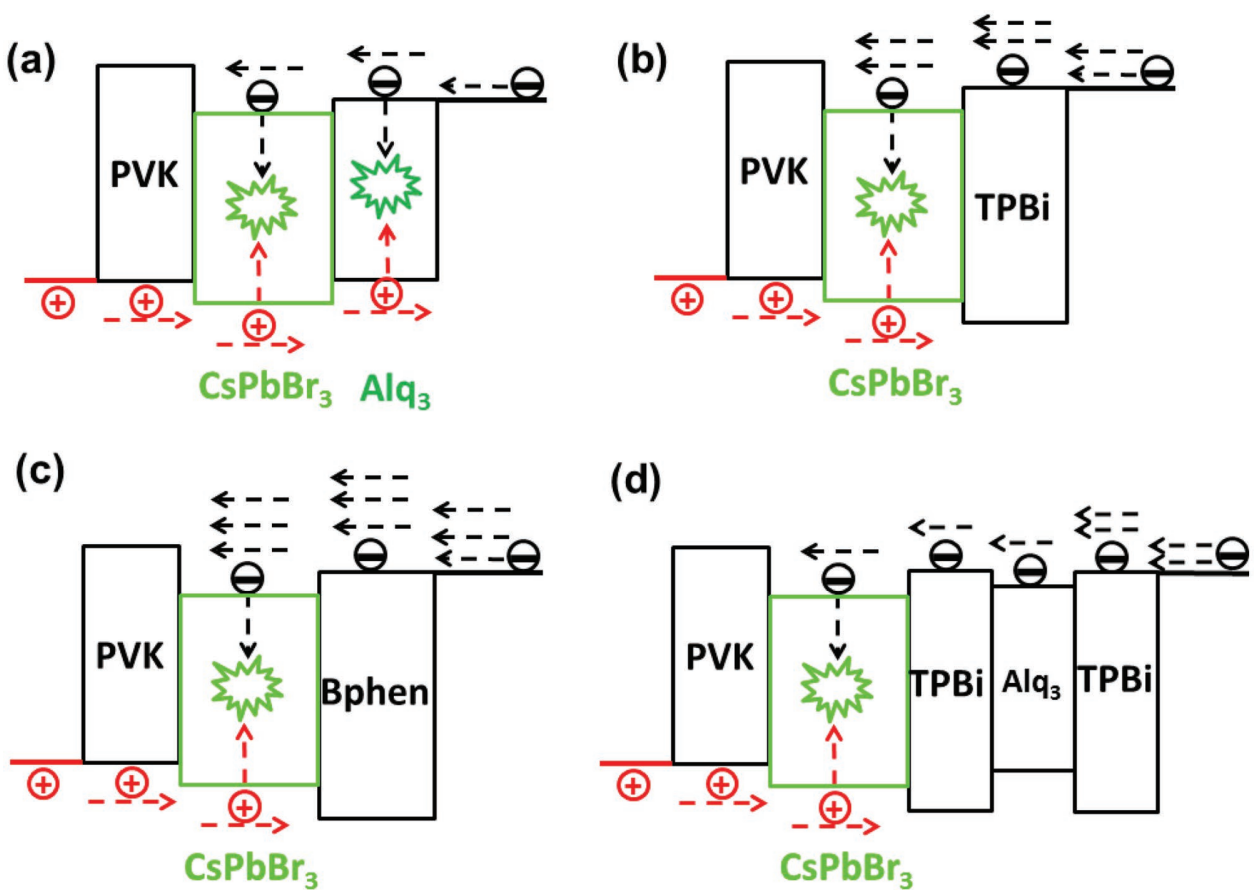

Figure 3. The working mechanisms of PeLEDs based on $\mathrm{CsPbBr}_{3}$ : a) Device G1, b) Device G2, c) Device G3, and d) Device G4. The red and black arrows represent the hole and electron transport, respectively. 
the electron mobility of $\mathrm{Alq}_{3}$ is lower than that of TPBi, the insertion of $\mathrm{Alq}_{3}$ can impede the electron transport, as shown in Figure 3d. In other words, the $\mathrm{Alq}_{3}$ reduces the number of electrons passing through it. As a result, a relatively appropriate number of electrons can reach the $\mathrm{CsPbBr}_{3}$ EML. Considering the electron mobility of $\mathrm{Alq}_{3}$ is almost equal to the hole mobility of PVK, the charge balance is more ideal in Device G4. On the other hand, TPBi can confine the hole transport. Therefore, the proposed strategy can simultaneously achieve the charge balance and charge confinement. To provide more direct evidence to support these analyses, electron-only devices have been fabricated. ${ }^{[45]}$ The architecture is ITO/zinc oxide $(\mathrm{ZnO}$, $40 \mathrm{~nm}) / \mathrm{CsPbBr}_{3}(20 \mathrm{~nm}) / \mathrm{ETLs}(35 \mathrm{~nm}) / \mathrm{Cs}_{2} \mathrm{CO}_{3}(1 \mathrm{~nm}) / \mathrm{Al}$ $(100 \mathrm{~nm})$, where ETLs are $\mathrm{Alq}_{3}$ (Device E1), TPBi (Device E2), Bphen (Device E3), and TPBi/Alq $/$ TPBi (Device E4). Due to the very deep VB of $\mathrm{ZnO}(\approx 7.6 \mathrm{eV}),{ }^{[26]}$ holes cannot be injected from the ITO anode $(4.7 \mathrm{eV}){ }^{[39]}$ As shown in Figure S5 (Supporting Information), because of the high electron mobility and good electron injection efficiency of Bphen, it is reasonable that Device E3 exhibits the highest current density among these devices. Besides, Device E1 shows the lowest current density owing to the low electron mobility of $\mathrm{Alq}_{3}$. Compared with Device E2, Device E4 exhibits lower current density due to the insertion of $\mathrm{Al}_{q_{3}}$, which can slow down the electron transport. Thus, the charge balance can be more ideal in Device G4. On the other hand, to further support the charge confinement of the new ETL TPBi/Alq $q_{3} / \mathrm{TPBi}$, a PeLED with the ETL $\mathrm{Alq}_{3}$ $(10 \mathrm{~nm}) / \mathrm{TPBi}(25 \mathrm{~nm})$ is developed, where other layers are the same as those of Device G4. As shown in Figure S6 (Supporting Information), this PeLED exhibits a broad EL spectrum, which is attributed to the participation of $\mathrm{Alq}_{3}$ emission since the ETL Alq $/$ TPBi cannot confine holes. Thus, the hole confinement is much better in Device G4 than using perovskite/Alq 3 /TPBi structure.

To further verify the above analyses, the EL performance of Device G4 has been measured. As shown in Figure 4, Device G4 exhibits a maximum EQE, CE, and PE of 1.43\%, $4.69 \mathrm{~cd} \mathrm{~A}^{-1}$, and $1.84 \mathrm{~lm} \mathrm{~W}^{-1}$, respectively, which are $191 \%, 192 \%$, and 211\% higher than those of Device G2 using the conventional ETL TPBi. Therefore, the proposed strategy can greatly enhance the efficiency of PeLEDs. Significantly, the EQE of Device G4 is 36 times higher than that of Device G3 with the high electron mobility Bphen ETL, further indicating the advantage of the proposed strategy. Such a surprising phenomenon that the device with low electron transport shows strikingly higher efficiency than the device with good electron transport has not been observed previously, which offers a deep insight into the effect of ETLs in nanocrystal all-inorganic PeLEDs. Besides, the EQE of Device G4 is higher than or comparable to those of recently reported representative performance levels of $\mathrm{CsPbBr}_{3}$ PeLEDs (e.g., 1.37\% for Cho's device, ${ }^{[46]} 1.49 \%$ for Zeng's device, ${ }^{[47]}$ and $\leq 0.93 \%$ for other devices ${ }^{[48-54]}$ ). In addition, the maximum luminance of Device G4 is $452 \mathrm{~cd} \mathrm{~m}^{-2}$, much higher than that of Device G2 with the conventional ETL TPBi (226 cd m²; Figure S7, Supporting Information). Moreover, Device G4 shows a pure green emission with the Commission International de I'Eclairage (CIE) coordinates of $(0.09,0.76)$, as shown in Figure 4d. Impressively, the FWHM of the EL emission is only $16 \mathrm{~nm}$, which is the narrowest value among all $\mathrm{CsPbBr}_{3}$ PeLEDs reported, to the best of our knowledge. The reproducibility of devices with different ETLs is very high. For example, over $80 \%$ of PeLEDs with the new ETL provide the EQE of $>1.2 \%$, as shown by the histograms of maximum EQE taken from 18 devices (Figure S8, Supporting
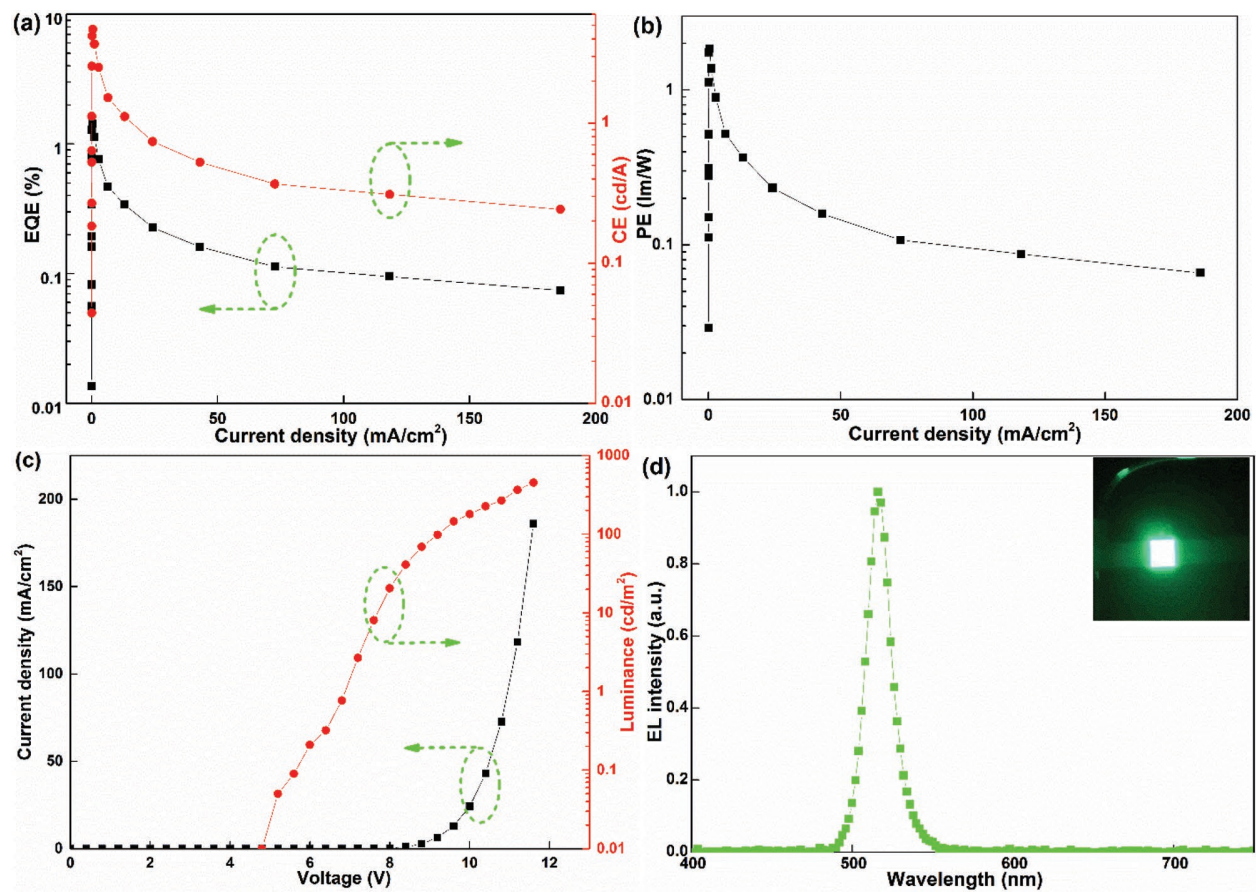

Figure 4. EL performance of Device G4. a) EQE and CE. b) PE. c) Current density and luminance. d) EL spectrum at 8.5 V. Inset: a photograph of Device G4 under bias. 
Information). In addition, the device operational stability is a major issue in PeLED fields. However, only very few works reported it, even for all-inorganic PeLEDs. Hence, it is urged that some stability data should be revealed, which is beneficial to deeply comprehend PeLEDs. The stability of PeLEDs under continuous operation at a constant voltage of $9 \mathrm{~V}$ has been evaluated in ambient air at room temperature, and we define the half-lifetime $\left(T_{50}\right)$ as the time for the luminance decreasing to $50 \%$ of the starting luminance $\left(L_{0}\right)$. As shown in Figure $S 9$ (Supporting Information), the $T_{50}$ of Device G4 is $460 \mathrm{~s}$, which is similar to previous all-inorganic PeLEDs (e.g., $600 \mathrm{~s}$ at $5 \mathrm{~V}$ in nitrogen filled glovebox ${ }^{[27]}$ ) despite that our test conditions are much more severe. On the other hand, the $T_{50}$ of Device G2 is only 150 s, which is $307 \%$ shorter than that of Device G4. Therefore, the better charge balance can enhance the stability, which is in agreement with the phenomenon reported in II-VI group metal chacogenide quantum-dot LEDs. ${ }^{[26]}$

Previously, Rogach and co-workers improved the devices efficiency by sandwiching additional layer between the EML and charge transport layers. ${ }^{[27,28]}$ Here, we have proposed another strategy to enhance the efficiency of all-inorganic nanocrystal PeLEDs, and the efficiency obtained here is much higher than that of their devices. ${ }^{[27,28]}$ Besides, by stepwise optimizing the emitting materials $\mathrm{CsPbBr}_{3}$, Zeng and co-workers achieved a 50-fold EQE improvement. ${ }^{[22]}$ From the perspective of device engineering, we have demonstrated that large EQE enhancement (36-fold) can also be achieved via adopting suitable ETLs. Therefore, the presented results may provide an alternative avenue to boost the efficiency of all-inorganic PeLEDs. Besides, since there is no report that ETL based on low electron mobility materials $\left(<1.0 \times 10^{-5} \mathrm{~cm}^{2} \mathrm{~V}^{-1} \mathrm{~s}^{-1}\right)$ can outperform ETL with high electron mobility materials $\left(>1.0 \times 10^{-5} \mathrm{~cm}^{2} \mathrm{~V}^{-1} \mathrm{~s}^{-1}\right)$ in all-inorganic PeLEDs, our strategy combining materials with low and high electron mobility can be an effective alternative. Hence, the findings may unlock a new opportunity that a class of materials with low electron mobility $\left(<1.0 \times 10^{-5} \mathrm{~cm}^{2} \mathrm{~V}^{-1} \mathrm{~s}^{-1}\right)$ are promising to achieve highly efficient PeLEDs. Moreover, our idea can be further validated by applying to other emitters. For example, by using a typical deep-red organic emitter tris(1phenylisoquinolinolato- $\left.C^{2}, N\right)$ iridium(III) $\left[\operatorname{Ir}(\mathrm{piq})_{3}\right],{ }^{[55,56]}$ an OLED with the EML Ir(piq) $3(0.5 \mathrm{~nm})$ is developed, where other layers are the same as those of Device G4 except for the EML. As shown in Figure S10 (Supporting Information), this OLED exhibits a maximum EQE of 3.24\%, which is among the highest $\operatorname{Ir}\left(\right.$ piq) ${ }_{3}$-based nondoped deep-red OLEDs. The CIE coordinates is $(0.68,0.32)$, which is very close to the National Television System Committee standard for red subpixels $(0.67,0.33)$.

In summary, a novel strategy has been proposed and implemented to improve the device engineering of nanocrystal allinorganic PeLEDs by incorporating $\mathrm{Alq}_{3}$ into TPBi to form a new ETL TPBi/Alq ${ }_{3} / \mathrm{TPBi}$, simultaneously achieving the charge balance and charge confinement. The green PeLED with the new ETL TPBi/Alq $/$ TPBi exhibits a maximum EQE, CE, and $\mathrm{PE}$ of $1.43 \%, 4.69 \mathrm{~cd} \mathrm{~A}^{-1}$, and $1.84 \mathrm{~lm} \mathrm{~W}^{-1}$, respectively, much higher than those of the PeLED with the most popular ETL TPBi. Remarkably, the efficiency is 36 times higher than that of PeLED using high electron mobility ETL. Also impressively, the FWHM of EL emission is only $16 \mathrm{~nm}$, the narrowest value among all achieved $\mathrm{CsPbr}_{3} \mathrm{PeLEDs}$ reported to date. The find- ings present a rational strategy for the device engineering of allinorganic PeLEDs.

\section{Supporting Information}

Supporting Information is available from the Wiley Online Library or from the author.

\section{Acknowledgements}

B.L. and L.W. contributed equally to this work. This research is supported by the National Research Foundation, Prime Minister's Office, Singapore, under its Competitive Research program (NRFCRP14-2014-03), its Investigatorship program (NRF-NRFI2016-08) and the Singapore Agency for Science, Technology and Research (A*STAR) SERC Pharos Program under Grant No. 15273 00025, Singapore Ministry of Education through the Academic Research Fund under Projects MOE2016-T2-1-054, Tier 1-RG105/16 and Tier 1- RG92/15, and China Scholarship Council ([2016]3100, No. 201608420137). H.V.D. gratefully acknowledges TUBA-GEBIP.

\section{Conflict of Interest}

The authors declare no conflict of interest.

\section{Keywords}

electron transport layer, light-emitting diodes, nanocrystals, perovskites

Received: February 19, 2018

Published online: March 22, 2018

[1] M. M. Lee, J. Teuscher, T. Miyasaka, T. N. Murakami, H. J. Snaith, Science 2012, 338, 643.

[2] J. Luo, J. H. Im, M. T. Mayer, M. Schreier, M. K. Nazeeruddin, N. G. Park, S. D. Tilley, H. J. Fan, M. Grätzel, Science 2014, 345, 1593.

[3] N. J. Jeon, J. H. Noh, W. S. Yang, Y. C. Kim, S. Ryu, J. Seo, S. I. Seok, Nature 2015, 517, 476.

[4] H. Zhu, Y. Fu, F. Meng, X. Wu, Z. Gong, Q. Ding, M. V. Gustafsson, M. T. Trinh, S. Jin, X. Y. Zhu, Nat. Mater. 2015, 14, 636.

[5] B. R. Sutherland, E. H. Sargent, Nat. Photonics 2016, 10, 295

[6] S. D. Stranks, H. J. Snaith, Nat. Nanotechnol. 2015, 10, 391.

[7] Z. K. Tan, R. S. Moghaddam, M. L. Lai, P. Docampo, R. Higler, F. Deschler, M. Price, A. Sadhanala, L. M. Pazos, D. Credgington, F. Hanusch, T. Bein, H. J. Snaith, R. H. Friend, Nat. Nanotechnol. 2014, 9, 687 .

[8] H. Cho, S.-H. Jeong, M.-H. Park, Y.-H. Kim, C. Wolf, C.-L. Lee, J. H. Heo, A. Sadhanala, N. Myoung, S. Yoo, S. H. Im, R. H. Friend, T.-W. Lee, Science 2015, 350, 1222.

[9] Y.-H. Kim, H. Cho, J. H. Heo, T.-S. Kim, N. Myoung, C.-L. Lee, S. H. Im, T.-W. Lee, Adv. Mater. 2015, 27, 1248.

[10] Y.-H. Kim, H. Cho, T.-W. Lee, Proc. Natl. Acad. Sci. USA 2016, 113, 11694.

[11] N. Wang, L. Cheng, R. Ge, S. Zhang, Y. Miao, W. Zou, C. Yi, Y. Sun, Y. Cao, R. Yang, Y. Wei, Q. Guo, Y. Ke, M. Yu, Y. Jin, Y. Liu, Q. Ding, D. Di, L. Yang, G. Xing, H. Tian, C. Jin, F. Gao, R. H. Friend, J. Wang, W. Huang, Nat. Nanotechnol. 2016, 10, 699. 
[12] L. Protesescu, S. Yakunin, M. I. Bodnarchuk, F. Krieg, R. Caputo, C. H. Hendon, R. X. Yang, A. Walsh, M. V. Kovalenko, Nano Lett. 2015, 15, 3692.

[13] G. R. Yettapu, D. Talukdar, S. Sarkar, A. Swarnkar, A. Nag, P. Ghosh, P. Mandal, Nano Lett. 2016, 16, 4838.

[14] A. Pan, B. He, X. Fan, Z. Liu, J. J. Urban, A. P. Alivisatos, L. He, Y. Liu, ACS Nano 2016, 10, 7943.

[15] J. Song, J. Li, X. Li, L. Xu, Y. Dong, H. Zeng, Adv. Mater. 2015, 27, 7162.

[16] Y. Wang, X. Li, J. Song, L. Xiao, H. Zeng, H. Sun, Adv. Mater. 2015, 27, 7101.

[17] S. Yakunin, L. Protesescu, F. Krieg, M. I. Bodnarchuk, G. Nedelcu, M. Humer, G. De Luca, M. Fiebig, W. Heiss, M. V. Kovalenko, Nat. Commun. 2015, 6, 8056.

[18] Y. Wang, X. Li, V. Nalla, H. Zeng, H. Sun, Adv. Funct. Mater. 2017, 27, 1605088

[19] G. Li, F. W. R. Rivarola, N. J. L. K. Davis, S. Bai, T. C. Jellicoe, F. de la Peña, S. Hou, C. Ducati, F. Gao, R. H. Friend, N. C. Greenham, Z.-K. Tan, Adv. Mater. 2016, 28, 3528.

[20] J. Pan, L. N. Quan, Y. Zhao, W. Peng, B. Murali, S. P. Sarmah, M. Yuan, L. Sinatra, N. M. Alyami, J. Liu, E. Yassitepe, Z. Yang, O. Voznyy, R. Comin, M. N. Hedhili, O. F. Mohammed, Z. H. Lu, D. H. Kim, E. H. Sargent, O. M. Bakr, Adv. Mater. 2016, 28, 8718.

[21] A. Swarnkar, A. R. Marshall, E. M. Sanehira, B. D. Chernomordik, D. T. Moore, J. A. Christians, T. Chakrabarti, J. M. Luther, Science 2016, 354, 92.

[22] J. Li, L. Xu, T. Wang, J. Song, J. Chen, J. Xue, Y. Dong, B. Cai, G. Shan, B. Han, H. Zeng, Adv. Mater. 2017, 29, 1603885.

[23] Y. Ling, Y. Tian, X. Wang, J. C. Wang, J. M. Knox, F. Perez-Orive, Y. Du, L. Tan, K. Hanson, B. Ma, H. Gao, Adv. Mater. 2016, 28, 8983.

[24] H.-C. Wang, W. Wang, A.-C. Tang, H.-Y. Tsai, Z. Bao, T. Ihara, N. Yarita, H. Tahara, T. Kanemitsu, S. Chen, R. S. Liu, Angew. Chem., Int. Ed. 2017, 129, 13838.

[25] X. Zhang, B. Xu, J. Zhang, Y. Gao, Y. Zheng, K. Wang, X. W. Sun, Adv. Funct. Mater. 2016, 26, 4595

[26] X. Dai, Z. Zhang, Y. Jin, Y. Niu, H. Cao, X. Liang, L. Chen, J. Wang, $X$. Peng, Nature 2014, 515, 96.

[27] X. Zhang, H. Lin, H. Huang, C. Reckmeier, Y. Zhang, W. C. H. Choy, A. L. Rogach, Nano Lett. 2016, 16, 1415.

[28] H. Huang, H. Lin, S. V. Kershaw, A. S. Susha, W. C. H. Choy, A. L. Rogach, J. Phys. Chem. Lett. 2016, 7, 4398.

[29] C. Wu, Y. Zou, T. Wu, M. Ban, V. Pecunia, Y. Han, Q. Liu, T. Song, S. Duhm, B. Sun, Adv. Funct. Mater. 2017, 27, 1700338.

[30] B. S. Mashford, M. Stevenson, Z. Popovic, C. Hamilton, Z. Zhou, C. Breen, J. Steckel, V. Bulovic, M. Bawendi, S. Coe-Sullivan, P. T. Kazlas, Nat. Photonics 2013, 7, 407.

[31] C. W. Tang, V. A. VanSlyke, Appl. Phys. Lett. 1987, 51, 913.
[32] S. Kim, H.-J. Kwon, S. Lee, H. Shim, Y. Chun, W. Choi, J. Kwack, D. Han, M. Song, S. Kim, S. Mohammadi, I. Kee, S. Y. Lee, Adv. Mater. 2011, 23, 3511.

[33] S. O. Yun, Y. Hwang, J. Park, Y. Jeong, S. H. Kim, B, I. Noh, H. S. Jung, H. S. Jang, Y. Hyun, S.-H. Choa, H. C. Ko, Adv. Mater. 2013, 25, 5626.

[34] P. Freederich, V. Comez, C. Sprau, V. Meded, T. Strunk, M. Jenne, A. Magri, F. Symalla, A. Colsmann, M. Ruben, W. Wenzel, Adv. Mater. 2017, 29, 1703505.

[35] S. Nau, C. Wolf, S. Sax, E. J. W. List-Kratochvil, Adv. Mater. 2015, 27, 1048.

[36] B. Hu, Y. Wu, Nat. Mater. 2007, 6, 985.

[37] K. J. Russell, T.-L. Liu, S. Cui, E. L. Hu, Nat. Photonics 2012, 6, 459.

[38] A. Droghetti, P. Thielen, I. Rungger, N. Haag, N. GroBmann, J. Stockl, B. Stadtmuller, M. Aeschlimann, S. Sanvito, M. Cinchetti, Nat. Commun. 2016, 7, 12668.

[39] Q. Wang, D. Ma, Chem. Soc. Rev. 2010, 39, 2387.

[40] B. Liu, H. Nie, X. Zhou, S. Hu, D. Luo, D. Gao, J. Zou, M. Xu, L. Wang, Z. Zhao, A. Qin, J. Peng, H. Ning, Y. Cao, B. Z. Tang, Adv. Funct. Mater. 2016, 26, 776.

[41] I.-W. Wu, P.-S. Wang, W.-H. Tseng, J.-H. Chang, C.-I. Wu, Org. Electron. 2012, 13, 13

[42] Y. Shirasaki, G. J. Supran, M. G. Bawendi, V. Bulovic, Nat. Photonics 2013, 7, 13

[43] N. C. Erickson, R. J. Holmes, Appl. Phys. Lett. 2010, 97, 083308.

[44] C. W. Tang, S. A. VanSlyke, Appl. Phys. Lett. 1987, 51, 913.

[45] M.-H. Park, S.-H. Jeong, H.-K. Seo, C. Wolf, Y.-H. Kim, H. Kim, J. Byun, J. S. Kim, H. Cho, T.-W. Lee, Nano Energy 2017, 42, 157.

[46] H. Cho, C. Wolf, J, S. Kim, H. J. Yun, S. S. Bae, H, Kim , J.-M. Heo, S. Ahn, T.-W. Lee, Adv. Mater. 2017, 29, 1700579.

[47] S. Zou, Y. Liu, J. Li, C. Liu, R. Feng, F. Jiang, Y. Li, J. Song, H. Zeng, M. Hong, X. Chen, J. Am. Chem. Soc. 2017, 139, 11443.

[48] X. Zhang, W. Wang, B. Xu, S. Liu, H, Dai , D. Bian, S. Chen, K. Wang, X. W. Sun, Nano Energy 2017, 37, 40.

[49] Q. V. Le, J. B. Kim, S. Y. Kim, B. Lee, D. R. Lee, J. Phys. Chem. Lett. 2017, 8,4140.

[50] X. Zhang, B. Xu, W. Wang, S. Liu, Y. Zheng, S. Chen, K. Wang, X. W. Sun, ACS Appl. Mater. Interfaces 2017, 9, 4926.

[51] Q. Shan, J. Li, J. Song, Y. Zou, L. Xu, J. Xue, Y. Dong, C. Huo, J. Chen, B. Han, H. Zeng, J. Mater. Chem. C 2017, 5, 4565.

[52] S. Wei, Y. Yang, X. Kang, L. Wang, L. Huang, D. Pan, Inorg. Chem. 2017, 56, 2596

[53] Q. V. Le, M. Park, W. Sohn, H. W. Jang, S. Y. Kim, Adv. Electron. Mater. 2017, 3, 1600448

[54] X. Du, G. Wu, J. Cheng, H. Dang, K. Ma, Y.-W. Zhang, P.-F. Tan, S. Chen, RSC Adv. 2017, 7, 10391

[55] B. Liu, L. Wang, D. Gao, M. Xu, X. Zhu, J. Zou, L. Lan, H. Ning, J. Peng, Y. Cao, Mater. Horiz. 2015, 2, 536.

[56] B. Liu, H. Tao, L. Wang, D. Gao, W. Liu, J. Zou, M. Xu, H. Ning, J. Peng, Y. Cao, Nano Energy 2016, 26, 26. 